\title{
Analytical and statistical methods to evaluate microsatellite allelic imbalance in small amounts of DNA
}

\author{
Robbert JC Slebos ${ }^{1}$, David M Umbach ${ }^{2}$, Courtney A Sommer ${ }^{1}$, Geoffrey A Horner ${ }^{1}$, \\ Jane Y Choi ${ }^{1}$ and Jack A Taylor ${ }^{1,3}$ \\ ${ }^{1}$ Laboratory of Molecular Carcinogenesis; ${ }^{2}$ Biostatistics Branch and ${ }^{3}$ Epidemiology Branch, National Institute \\ of Environmental Health Sciences, National Institutes of Health, Research Triangle Park, NC, USA
}

\begin{abstract}
Microsatellite analysis is a powerful tool for the assessment of genetic instability and loss of heterozygosity in cancer cells. However, most human tumors harbor significant numbers of normal cells, which may contribute to false-negative results. Recent techniques based on fluorescently labeled primers and semiautomated capillary electrophoresis of polymerase chain reaction (PCR) products allow a reliable quantitative assessment of (PCR) products while requiring very small numbers of cells. We report a highly sensitive protocol for the semiautomated analysis of allelic imbalance based on time-release PCR and capillary electrophoresis. With this protocol, as few as 100 cells can be used to reliably assess allelic imbalance (AI) in DNA samples. Using a panel of seven microsatellite markers, we determined allelic variation in a large set of heterozygous lymphocyte DNA samples and examined the use of different statistical analysis techniques. Using these statistical approaches, we describe a calibration method to evaluate Al from microsatellite results. Using a simple formula, cutoff points at preset confidence levels are used to decide whether allelic imbalance exists in a given sample at the loci under investigation. Our method allows the reliable detection of Al with very small amounts of DNA, and is sufficiently quantitative to assess allelic ratios in nonclonal tissue specimens.
\end{abstract}

Laboratory Investigation (2004) 84, 649-657, advance online publication, 29 March 2004; doi:10.1038/labinvest.3700076

Keywords: microsatellite; loss of heterozygosity; allelic imbalance; time-release PCR; tissue microdissection

Abnormal chromosomes, or aneuploidy, is one hallmark of cancer cells. ${ }^{1}$ This chromosomal imbalance is characterized by losses or gains of chromosomal regions, duplication of chromosomes, and the amplification of DNA. Whether aneuploidy is the cause or effect of carcinogenesis is still unclear, although some of the genetic changes do affect oncogenes and tumor suppressor genes. Inactivating mutations in tumor suppressor genes are frequently associated with loss of the nonmutated allele of the gene, called loss of heterozygosity (LOH). In mixtures of normal and tumor cells, LOH may only be detectable as an imbalance between the detected amounts of each of the two alleles (allelic imbalance, AI). Measures of AI may be further complicated by other chromosomal abnormalities, such as duplications, that may lead to altered allele ratios.

Correspondence: JA Taylor, Epidemiology Branch, National Institute of Environmental Health Sciences, Maildrop A3-01, PO Box 12233, Research Triangle Park, NC 27709, USA.

E-mail: taylor@niehs.nih.gov

Received 22 May 2003; revised 26 September 2003; accepted 01 October 2003; published online 29 March 2004
The analysis of microsatellites in DNA from normal or tumor cells has proven a powerful tool to determine genetic changes associated with cancer development. Microsatellites consist of repetitive DNA sequences, with repeat units typically ranging from one to five nucleotides (for review see Umar and $\mathrm{Kunkel}^{2}$ ). These simple sequences can pose difficulties for cellular DNA replication and repair mechanisms. As a result, microsatellites are highly variable within populations, and mutations in microsatellites are more common than those in nonrepetitive DNA. Because of their high mutability, many individual alleles of a given microsatellite may exist in the human population. This variation translates into a high probability that different length alleles exist when analyzing different individuals. For this reason, microsatellites at or near tumor-associated genes have been used to localize these genes and to analyze genetic abnormalities in cancer cells. For example, LOH at specific chromosomal loci has been used to study the sequence of events associated with carcinogenesis and to determine the clinical behavior of different tumors. ${ }^{3-5}$ 
Because LOH analysis depends on the absence of signal for one of the two alleles, it is very sensitive to contaminating normal tissue in the DNA specimen. Contamination is almost always a problem since primary tumors contain a mixture of neoplastic and non-neoplastic cells. These non-neoplastic cells can be stromal cells, infiltrating lymphocytes, endothelial cells, or others. The DNA contribution of these cells can obscure the molecular abnormalities found in neoplastic cells. Recent developments in microdissection of neoplastic cells have provided a reliable way to obtain almost pure populations of cells. However, the capability to analyze fewer and fewer cells using sensitive molecular techniques creates new challenges in the assessment and interpretation of the data. The most important of these are the question of whether the sample assayed is representative of the tissue of interest, the technical challenges of analyzing minute amounts of DNA, and the statistical analysis of the resulting measurements. Tissue representation is an issue whenever one aims to generalize findings on a small tissue sample or biopsy to the diseased organ or to the presence, or absence of a disease. These questions are not new, but with the capability to analyze smaller numbers of cells obtaining an accurate representation of the targeted tissue becomes increasingly important. In some cases, the assumption of an exclusive mixture of heterozygous and nonheterozygous cells does not hold. Apparently, normal cell populations may contain a number of cells with abnormal DNA content and/ or $\mathrm{LOH}$ and the proportion of such cells may vary within the sample set. In the case of bronchial epithelium, it was proposed that patches of cells are the result of clonal outgrowth and that each of these patches of cells may carry different genetic mutations. ${ }^{6}$ An accurate description of the level of AI is critical under these conditions. Over the last decade, many of the conditions for the analysis of $\mathrm{AI}$ and LOH in small amounts of DNA have been investigated using traditional radioactive labeling and gel electrophoresis. $^{7-9}$ The use of automated capillary electrophoresis to analyze fluorescently labeled DNA fragments is relatively new, however, with only a few validation studies have been reported..$^{10-12}$

The goal of the current study was to develop a reliable protocol optimized for sensitivity and accuracy. The new protocol employs a one-step DNA extraction, followed by a single time-release amplification reaction with analysis of the fluorescently labeled polymerase chain reaction (PCR) product by capillary electrophoresis. This approach allowed us to reduce the number of processing steps, thereby minimizing the risk of contamination. The current protocol can detect single molecules of DNA obtained from frozen cells. It is highly reproducible and can reliably detect $\mathrm{AI}$ in as few as 100 cells. We propose a statistical approach based on transformation of allele ratios that can be used to estimate the extent of $\mathrm{AI}$ in a given sample and to decide (with a specified level of confidence) whether AI is actually present.

\section{Materials and methods}

\section{DNA Isolation and Quantitation}

Human lymphocyte DNA samples used in this study were collected as part of a clinical study on the early detection of lung cancer in high-risk patients. This study was approved by the Institutional Review Boards of the University of North Carolina at Chapel Hill and of the National Institute of Environmental Health Sciences. Mononucleated blood cells were isolated within $24 \mathrm{~h}$ of phlebotomy using Vacutainer CPT tubes (Beckton-Dickinson, Franklin Lakes, NJ, USA) as recommended by the manufacturer and kept in a buffered $15 \%$ ethanol solution at $4^{\circ} \mathrm{C}$ until DNA isolation. DNA was isolated from these cells using a protocol that is commonly used for both frozen and fixed tumor specimens. ${ }^{13}$ Briefly, approximately $10^{5}$ cells were incubated in $100 \mu \mathrm{l}$ of lysis buffer $(0.2 \%$ Tween-80, $100 \mu \mathrm{g}$ Proteinase $\mathrm{K} / \mathrm{ml}$, in $10 \mathrm{mM}$ Tris-HCl $\mathrm{pH} 7.5$ and $1 \mathrm{mM}$ EDTA) for $2 \mathrm{~h}$ at $56{ }^{\circ}$ C. ${ }^{14}$ The DNA preparations were kept at $-20^{\circ} \mathrm{C}$ until analysis. A volume of $1 \mu \mathrm{l}$ of the DNA solution was used to quantify the DNA with PicoGreen DNA reagent (Molecular Probes, Eugene, OR, USA) as indicated by the manufacturer. This quantitation is based on SYBRgreen, which specifically binds double stranded DNA and allows the detection of picogram quantities of DNA.

\section{Time-release PCR}

Microsatellite analysis was performed with the DNA amounts as indicated for each experiment using a modification of time-release PCR as described previously. ${ }^{15}$ DNA preparations were diluted and used directly in a $50 \mu \mathrm{l}$ amplification reaction using TaqGold DNA polymerase (Applied Biosystems, Foster City, CA, USA). TaqGold is a chemically modified form of Thermus aquaticus DNA polymerase that requires activation by heat for full activity. Time-release of TaqGold DNA polymerase activity is achieved by eliminating the heat activation step at the start of the protocol. These adaptations result in a slow release of polymerase activity during the initial phases of the PCR, when limited amounts of target DNA are available. These properties allow the amplification of a single molecule to proceed to quantities that are readily detectable by capillary electrophoresis using fluorescent dyes.

For the current study, a typical reaction contained 10-150 pg of DNA, amplification buffer with 1$3 \mathrm{mM} \mathrm{MgCl}_{2}, 200 \mu \mathrm{M}$ of each dNTP, $10 \mathrm{pmol}$ of each of the deoxyoligonucleotides and $2.5 \mathrm{U}$ of TaqGold in a volume of $50 \mu \mathrm{l}$. Optimization for the $\mathrm{MgCl}_{2}$ concentration was performed for each microsatellite marker at similarly low concentrations of DNA. The 
deoxyoligonucleotide primers were labeled with one of three fluorescent dyes, FAM, TET or HEX (Applied Biosystems, Foster City, CA, USA). The amplification schedule was identical for all microsatellites: a total of 60 cycles with a denaturation step of $30 \mathrm{~s}$ at $94^{\circ} \mathrm{C}$, an annealing step of $30 \mathrm{~s}$ at $55-60^{\circ} \mathrm{C}$, and a DNA synthesis step of $1 \mathrm{~min}$ at $72^{\circ} \mathrm{C}$, with a final extension for $5 \mathrm{~min}$ at $72^{\circ} \mathrm{C}$. A list of microsatellite markers used in this study is shown in Table 1. Recent experience has shown that a total of 50 amplification cycles is sufficient when analyzing the PCR products with the newer ABI 3100 capillary electrophoresis equipment.

\section{Analysis of PCR Products}

The amplification products were diluted 1 to 50 in deionized formamide and denatured by heating to $95^{\circ} \mathrm{C}$ for 3 min prior to loading on an ABI 310 DNA analyzer (Applied Biosystems, Foster City, CA, USA). A size marker standard was included with each run using TAMRA as a fluorescent dye (TAMRA 500, Applied Biosystems, Foster City, CA, USA). The results were analyzed with Genescan software using the peak area (area under the curve) as primary outcome measure. The area under the highest peak was chosen for analysis with markers that demonstrated DNA polymerase slippage artifacts. There were no substantial differences between the use of peak area or peak height as the outcome measure.

\section{Results}

\section{Time-release PCR}

We employed time-release PCR to analyze several different microsatellite markers that are of interest for lung carcinogenesis. We show here that this method is capable of amplifying amounts of DNA that are equivalent to single alleles present in the reaction. In addition, we investigated the reproducibility of allele ratios under amplification conditions that included DNA amounts ranging from the equivalent of just a few cells to hundreds of cells. Based on the results of these experiments we propose a statistical analysis procedure using allele ratios or allele fraction to assess $\mathrm{AI}$ in test samples. We also indicate how measures of AI may be used to calculate the proportion of cells exhibiting $\mathrm{LOH}$ in a mixed sample where some cells are heterozygous and some are hemizygous at the target locus.

The amplification procedure we developed is based on time-release PCR, which omits the initial heat activation step that is recommended for AmpliTaq Gold polymerase. ${ }^{15}$ Thus, enzyme activity is slowly released with less activity present at the initial cycles when only small amounts of DNA are present. The assumption is that this reduces the amount of nonspecific priming during the initial phases of the PCR. In addition, the Taq polymerase is exposed to less time at denaturing temperatures, so polymerase activity is still present even after 60 cycles (data not shown). The optimal conditions for each of the microsatellite markers were determined using $1 \mathrm{ng}$ of DNA (equivalent of about 170 cells, assuming 6 pg DNA/cell) using the time-release PCR protocol.

\section{Sensitivity and Allelic Representation Using Time-release PCR}

When amplifying amounts of DNA that approach single allele quantities per PCR vial, the distribution of molecules in separate vials becomes a chance event. Thus, results obtained from a set of independent PCR amplifications provide evidence for the sensitivity of the reaction and can verify that low amounts of DNA were indeed present. We tested these principles by PCR amplification of the marker D3S1298 in 10 independent vials containing the DNA equivalent of on average two diploid lymphocyte cells per vial. Representative examples of these types of amplifications are shown in Figure 1. Two of the 10 reactions did not show any product, while four showed only one of the two different alleles and another four showed both alleles. The allele ratios for these last four reactions were 1.01, 1.02, 1.93, and 0.59 , respectively. These results are consistent with a DNA concentration that is close to just a few molecules present per PCR vial and demonstrate the extreme sensitivity of time-release PCR when coupled with fluorescent detection by capillary electrophoresis.

Table 1 Overview of microsatellite markers

\begin{tabular}{lccccc}
\hline Microsatellite marker & Chromosomal location & Repeat unit & $\mathrm{N}$ & Average allele fraction (s.d.) & Average log (allele ratio) (s.d.) $^{2}$ \\
\hline D3S1300 & 3p14 & CA & 24 & $0.577(0.047)$ & $0.455(0.283)$ \\
D3S4103 & 3p14 & AAT & 29 & $0.588(0.054)$ & $0.525(0.336)$ \\
D3S1298 & 3p22-24.2 & CA & 21 & $0.620(0.054)$ & $0.721(0.368)$ \\
D3S1573 & 3p21.2 & CA & 21 & $0.603(0.040)$ & $0.607(0.244)$ \\
D9S171 & 9p21 & CA & 27 & $0.597(0.050)$ & $0.574(0.320)$ \\
IFNA & 9p22 & CA & 21 & $0.600(0.022)$ & $0.586(0.130)$ \\
TP53 & AAAAT 13 & 9 & $0.603(0.044)$ & $0.610(0.271)$
\end{tabular}


One important issue when dealing with the extremely small amounts of DNA, for instance obtained with microdissection techniques, is how well the original cell population of interest is represented by the DNA sample at hand. ${ }^{16}$ The lower the amount of DNA in the PCR, the greater the risk for allele ratios that are abnormal due to a chance distribution of alleles in the reaction. To examine how much DNA is needed to stabilize this source of variation, we analyzed marker D9S171 using starting amounts of a heterozygous DNA that were equivalent to $250,125,50,25,5$, and 0.5 cells with eight independent reactions per DNA level (Figure 2). The allele ratios in this experiment became more variable with decreasing DNA amounts. With the lowest DNA amount $(0.5$ cell equivalent) two reactions did not amplify, four
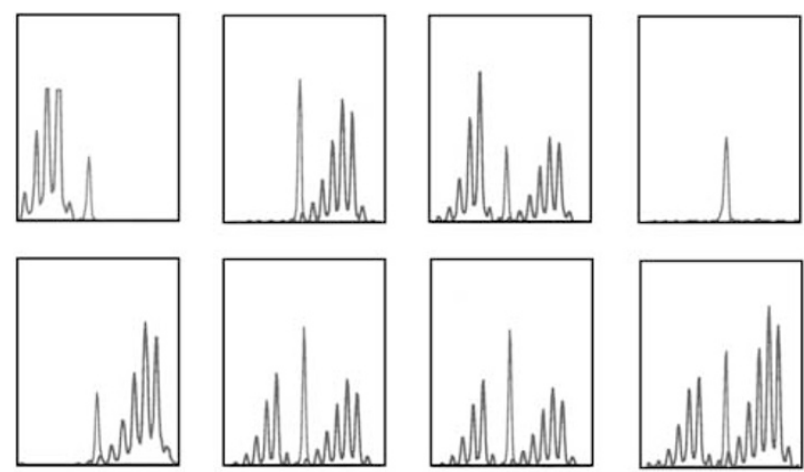

Figure 1 Example of PCR analysis using small amounts of DNA. Eight representative results are shown for the microsatellite marker D3S1298. Two distributions of allele sizes are shown (with PCR slippage artifacts) with a size standard peak in the middle. DNA quantities of approximately one genome equivalent of DNA per PCR vial were used.

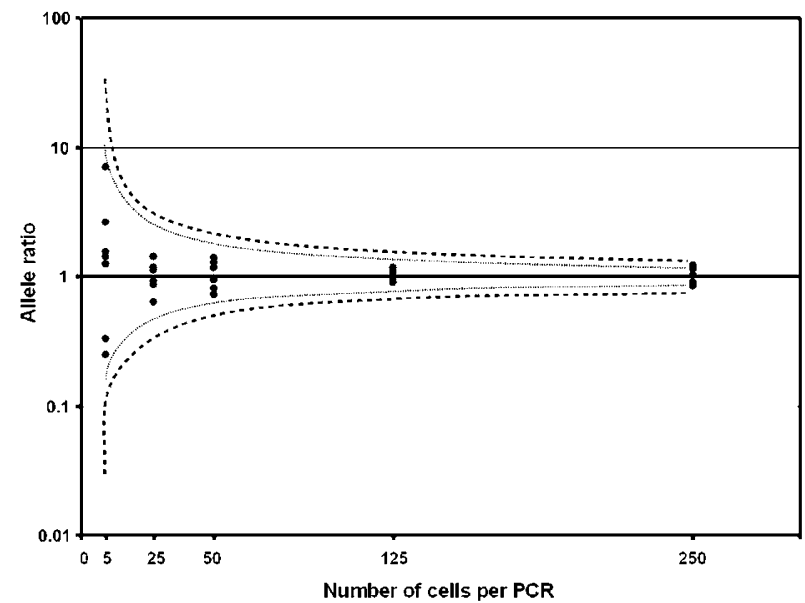

Figure 2 Distribution of allele ratios obtained with different amounts of starting DNA. The variability increases with smaller amounts of DNA in the PCR, and this variation becomes sufficiently large at cell equivalents of five or fewer cells to resemble PCR results normally obtained when allelic imbalance is present. Indicated are the 95 and $99 \%$ confidence intervals around the measurements. showed only one of the two alleles, while the remaining two had allele ratios of 0.99 and 1.03, respectively. Thus, the DNA equivalent of a minimum of about 100 cells is required for a full representation of both alleles in the analysis.

\section{Quantitative relationships among measures of Al and of $\mathrm{LOH}$}

Although AI is measured by our protocol, one is often interested in estimating the proportion of cells with $\mathrm{LOH}$ in a mixed sample. Allele ratio and allele fraction both measure AI, and either measure can easily be calculated from the other. In addition, under certain assumptions about the mixture of cells in a sample, the fraction of cells in the mixture that exhibit LOH can be calculated from either measure of AI.

First, we establish some notation. Suppose a tissue sample contains a mixture of diploid cells where some cells are heterozygous (AB, for example) at a particular locus and the remaining cells have $\mathrm{LOH}(\mathrm{A}-$, say) at that locus. Let $\pi$ be the fraction of A- cells in the mixture, that is, the fraction with $\mathrm{LOH}$. Let $f_{\mathrm{A}}$ and $f_{\mathrm{B}}$ denote the fraction of alleles in the DNA from the tissue sample that are A or B, respectively. Of course, $f_{\mathrm{B}}=1-f_{\mathrm{A}}$. The way that we have defined terms requires that $f_{\mathrm{A}} \geq f_{\mathrm{B}}$ or, equivalently, that $f_{\mathrm{A}} \geq \frac{1}{2}$, because only $\mathrm{B}$ alleles are lost. Also, let $R$ denote the allele ratio, that is, the ratio of the number of copies of allele $B$ to the number of copies of allele A (note that the allele with the smaller fraction is in the numerator so $R \leq 1$ in this notation).

Next, we show how to calculate one measure of AI from the other. To calculate $R$ from $f_{\mathrm{A}}$, notice that $R$ can be expressed as $f_{\mathrm{B}} / f_{\mathrm{A}}$. Thus, $R=\left(1-f_{\mathrm{A}}\right) / f_{\mathrm{A}}$. Solving this equation for $f_{\mathrm{A}}$ yields: $f_{\mathrm{A}}=1 /(R+1)$. These transformations from $f_{\mathrm{A}}$ to $R$ and vice versa apply to any complex mixture of multiple cell types that might exhibit AI.

Finally, consider calculating $\pi$, the proportion of cells in the tissue sample that exhibit $\mathrm{LOH}$, from measures of AI determined experimentally, assuming the simple mixture of two cell types described above. With some number $N$ of cells in the sample, one has $\pi N$ copies of A from the A-cells and $(1-\pi)$ $N$ copies of A from the AB cells, for a total of $N$ copies of A. With the same number of cells, one has only $(1-\pi) N$ copies of B from AB cells. Thus, the total number of alleles in the mixture is $N+(1-\pi)$ $N=(2-\pi) N$, somewhat less than the $2 N$ that one would have if all cells were heterozygous (that is, if $\pi=0)$. Consequently, the fraction of alleles in the mixture that are A is $f_{\mathrm{A}}=N /((2-\pi) N)=1 /(2-\pi)$. Solving this equation above for $\pi$ gives: $\pi=2-\left(1 / f_{\mathrm{A}}\right)$. Thus, the proportion of cells with $\mathrm{LOH}$ in a mixture can be calculated from the allele fraction. In particular, if $f_{\mathrm{A}}=\frac{1}{2}$ then $\pi=0$; that is, if half the alleles are allele A then there are no cells with $\mathrm{LOH}$ 
in the mixture. A similar argument shows that the fraction of alleles in the mixture that are $\mathrm{B}$ is

$$
f_{\mathrm{B}}=\frac{(1-\pi) N}{(2-\pi) N}=\frac{1-\pi}{2-\pi}
$$

Since $R=f_{\mathrm{B}} / f_{\mathrm{A}}$, substituting for $f_{\mathrm{A}}$ and $f_{\mathrm{B}}$ from the equations above gives $R=1-\pi$ or $\pi=1-R$. That is, the proportion of cells with $\mathrm{LOH}$ in the mixture can be calculated directly from the ratio of alleles (defined so that ratio is less than one).

\section{A Calibration Approach to Detecting AI}

We propose a statistical approach based on linear calibration for deciding whether an individual sample exhibits AI. Let $X$ denote the true allele ratio in a tissue with two alleles at a given locus. If the allele ratio differs from one, the tissue exhibits AI. Let $Y$ denote the estimated allele ratio measured by time-release PCR and fluorescent capillary electrophoresis. Suppose that one can find a oneto-one transformation of the allele ratio, say $T$, so that $T(Y)=\beta_{0}+\beta_{1} T(X)+\varepsilon$ (where $\varepsilon$ is a term for random error). Given such a calibration curve, one could estimate confidence bounds for the true allele ratio $X$ for any observed allele ratio of $Y$. If those confidence bounds failed to contain the null value one, one would declare that the observed $Y$ arose from a sample with AI. The standard linear calibration analysis assumes that the random errors in the calibration model have a constant variance regardless of the value of $T(X)$ and that they are approximately normally distributed. ${ }^{17}$ Consequently, we sought transformations of the allele ratio that appear consistent with those assumptions.

\section{Evaluation of Transformations of Allelic Ratio}

We used a mixture experiment to investigate the properties of possible transformation curves. Two homozygous lymphocyte DNA samples, each with different size alleles for marker IFNA, were mixed in proportions ranging from 0 to 1 (ie, 0 and $100 \%$ ) with a spacing of $0.1(10 \%)$. To this end, stock solutions of $30 \mathrm{pg} / \mu \mathrm{l}$ were created of each of the two DNA samples and different amounts of these stock solutions were mixed according to the indicated proportions to a total count of $600 \mathrm{pg}$ per PCR tube (the DNA equivalent of approximately 100 diploid cells). This low count was chosen to reflect the actual conditions under which the test specimens would be measured. The allele ratios in each mixing proportion were measured in four independently amplified subsamples using capillary electrophoresis. Ideally, a calibration curve in this context would exhibit an intercept of zero and a slope of one. Small variations in assessing the true DNA concentration in our lymphocyte DNA preparations made it difficult to create stock solutions containing precisely the same amount of DNA from each of the two DNA samples, so that the putatively equal amounts were likely somewhat discrepant. This problem would lead to a discrepancy between the nominal and the true allele ratios in a nominal 1:1 mixture. In addition, as mentioned elsewhere, the smaller allele generally had higher signal intensities in our measurements. These two features suggest that an intercept derived from our mixing experiment would be biased away from zero. On the other hand, the process that created the ladder of allele fractions from those initial putatively equal-concentration solutions should allow the spacing of the nominal mixing proportions to be more accurate, particularly for a logarithmic transformation of allele ratios. Consequently, we expected slopes to be near one.

We evaluated three potential transformations, where $y$ is a ratio between zero and infinity: allele ratio itself $(T(y)=y)$, the base 2 logarithm of the allele ratio $\left(T(y)=\log _{2}(y)\right.$, and the allele fraction $(T(y)=y /(1+y))$. As expected, for the preset mixing proportions 0 and 1 (allele ratios 0 and infinity), the measurement always resulted in allele fractions as 0 and 1, respectively. We ignored those two mixing proportions when evaluating the transformations. Each of the three transformations showed a reasonably linear relationship. The allele ratio was markedly heteroscedastic so we did not consider that transformation further. Both the logarithm of the allele ratio and the allele fraction exhibited the nearly constant variance across the range of mixing proportions in our experiment, although the allele fraction showed an expected tendency for the variance to decrease at mixing proportions nearer zero or one (Figure 3). The observed values corresponding to a preset allele fraction of $60 \%$ (with a $\log _{2}$ (allele ratio) of 0.585 ) deviate from the expected line, a discrepancy that we attribute to a possible pipetting error in establishing that mixture. Intercepts were smaller than zero $(P=0.08$ and $P<0.001$ for allele fraction and $\log _{2}$ (allele ratio), respectively); and the slopes did not differ significantly from one $(P=0.72$ and 0.18 for allele fraction and $\log _{2}$ (allele ratio), respectively). Thus, our mixing experiment suggests that either allele fraction or $\log _{2}$ (allele ratio), but not allele ratio, may be reasonable candidates for implementing a calibration approach to detect allelic imbalance. We prefer $\log _{2}$ (allele ratio) to allele fraction because the variance of the latter must decrease at extreme mixing proportions, contradicting a needed assumption.

\section{Deciding whether a sample exhibits allelic imbalance}

Although the results of the mixture experiment can be used to examine the properties of various transformations, including whether the expected slope is one, they cannot be used to estimate the 

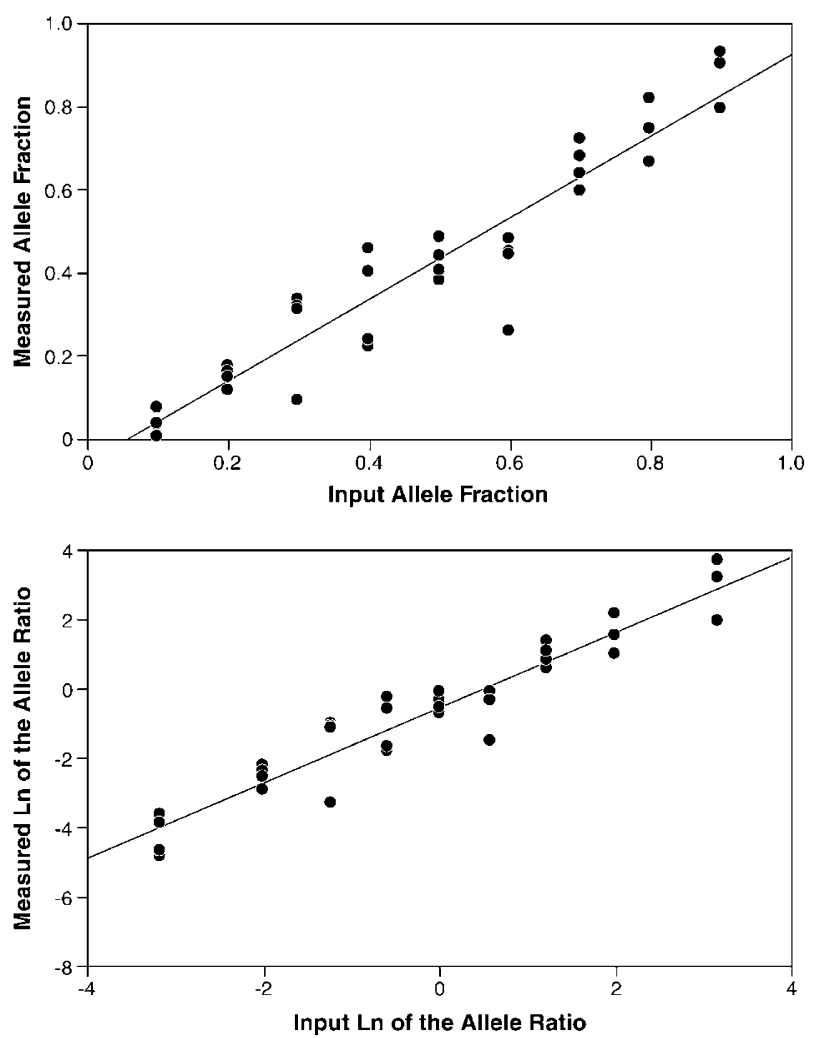

Figure 3 Evaluation of transformations of allele ratios for use in linear calibration inference about AI. The $x$-axis is the transformed value of the preset allele ratio in the sample; the $y$-axis is the transformed value of the observed allele ratio measured with capillary electrophoresis. All observed values follow expected values closely except the $60 \%$ input DNA (see text). Top panel, allele fraction; bottom panel, $\log _{2}$ (allele ratio).

calibration curve itself for two reasons. First, the expected bias in the intercept would lead to bias in the calibration inference. Second, the variation around the calibration line based on our artificially constructed 'heterozygotes' may differ from the variation that would be seen between actual heterozygous subjects when studying allelic imbalance, leading to improper confidence interval width. To alleviate these two difficulties, we propose a calibration procedure that relies on DNA from lymphocytes, a tissue that is unlikely to have allelic imbalance when, say, lung tissue is the object of study. Typically, the lymphocytes would come from the same individuals whose lung tissue is under study. Our procedure can use either the logarithm of the allele ratio or the allele fraction, and assumes that the true slope of the calibration line is one.

Data from the heterozygous lymphocytes without allelic imbalance allow us to estimate only a single point on the calibration curve, namely, the point where the allele ratio is $1: 1$ so that its logarithm is zero or the allele fraction is 0.5 . Under the credible assumption that the slope is one, however, the curve can then be estimated for any possible value of the allele ratio. Let $T_{\mathrm{i}}=T\left(Y_{i}\right)$ for $i=1,2,3, \ldots, n$ denote the

transformed version of the allele ratio, either the base 2 logarithm of the allele ratio or the allele fraction, measured in lymphocytes from the $i$ th heterozygous subject. Let $\bar{T}=1 / n \sum T_{i}$ be the average of the $T_{i}$, and let

$$
s=\sqrt{1 /(n-1) \sum\left(T_{i}-\bar{T}\right)^{2}}
$$

be the estimated standard deviation of the $T_{i}$. Under this notation, $T(1: 1)=0$ for the $\log _{2}$ (allele ratio) and $T(1: 1)=0.5$ for the allele fraction. Assuming a slope of one, take the estimated calibration curve to be $T(Y)=\bar{T}-T(1: 1)+T(X)$. Based on this estimated calibration curve, given any noncalibration observation (ie, one on the tissue of interest rather than on lymphocytes) denoted $T\left(Y^{*}\right)$, one can estimate the unknown true value $T\left(X^{\star}\right)$ as

$$
T\left(X^{*}\right)=T\left(Y^{*}\right)-\bar{T}+T(1: 1)
$$

where the expression $-\bar{T}+T(1: 1)$ corrects for the potential bias in the intercept mentioned earlier. The $(1-\alpha) \%$ confidence limits for $T\left(X^{*}\right)$ can be estimated using

$$
T(Y *)-\bar{T}+T(1: 1) \mp t_{n-1}^{\alpha} s \sqrt{1+\frac{1}{n}} .
$$

where $t_{n-1}^{\alpha}$ is the $(1-\alpha / 2)$ percentile of the Student's $t$ distribution with $n-1$ degrees of freedom. If the null value $T(1: 1)$ is outside these confidence limits, then one concludes with $(1-\alpha) \%$ confidence that the sample exhibits allelic imbalance. The validity of this confidence statement relies on the assumption that the $T_{i}$ follow a normal distribution, at least approximately.

\section{Normal Lymphocyte Allele Ratios for Seven Microsatellite Markers}

For each of the seven markers in this study, we assessed the allele ratios separately on two aliquots of lymphocytes from each subject and averaged the two transformed values from each subject to form a single reading. The averages of allele fractions or of $\log _{2}$ (allele ratios) across a number of DNA samples were similar across the seven markers (Table 1). For example, average allele fraction ranged from 0.577 to 0.620 , with standard deviations between 0.022 and 0.054

To illustrate the calculations for AI, we use the $\log _{2}$ (allele ratio) data from the marker IFNA. For the 21 subjects, the average $\log _{2}$ (allele ratio) had a mean of 0.586 and a standard deviation of 0.130 . Suppose we seek to decide whether an unknown sample with an observed average $\log _{2}$ (allele ratio) (based on two measurements) of 0.116 exhibits AI with $99 \%$ confidence. A point estimate for the true $\log _{2}$ (allele ratio $)$ is $-0.470(=0.116-0.586+0.0)$ with $99 \%$ confidence limits $(-0.849,-0.092)$. Since this interval does not cover 0.0, one would declare that the sample does exhibit AI at the 99\% confidence level. If one increased the desired confidence level to 
$99.9 \%$, the confidence interval becomes $(-0.982$, 0.042). This interval covers 0.0 so the sample fails to exhibit AI at the more stringent confidence level. Now, a $\log _{2}$ (allele ratio) of -0.470 corresponds to an allele fraction of 0.42. Assuming a mixture of heterozygous (normal) and hemizygous (having $\mathrm{LOH}$ ) cells, we can calculate the proportion of cells with $\mathrm{LOH}(\pi)$ in this example as using $f_{\mathrm{A}}=1-0.42$ (since $f_{\mathrm{A}}$ needs to be larger than $\frac{1}{2}$ ) so that $\pi=2-1$ / $0.58=0.28$. The proportion of cells with $\mathrm{LOH}$ in this example is $28 \%$. The observed $\log _{2}$ (allele ratio) of 0.116 in this example corresponds to an observed allele fraction of 0.52. Carrying out all the calculations in allele fraction scale would lead to slightly different confidence intervals (when the endpoints are transformed to a common scale) but the same conclusions about the existence of AI. Although conclusions about AI are not guaranteed to be exactly the same in the two scales; most often they will be.

\section{Discussion}

Microsatellite analysis has an important role in the assessment of genomic instability and mutation associated with carcinogenesis. Almost all tumors harbor gross chromosomal abnormalities as well as specific changes in and around cancer-associated genes. Various areas of $\mathrm{LOH}$ are also found in histologically normal bronchial epithelium, especially from patients with a history of exposure to tobacco smoke. ${ }^{18-20}$ Highly polymorphic microsatellites provide an informative and sensitive tool to analyze and characterize these changes. Traditionally, microsatellite markers were determined by radioactive labeling of PCR products followed by gel electrophoresis and autoradiography. The development of fluorescent dyes that can be analyzed in automated systems now allow for a more sensitive and a more quantitative characterization of microsatellite PCR products. In addition, novel microdissection techniques such as LCM can be used to obtain virtually pure cell populations from clinical samples that can be admixtures of many different cell types. ${ }^{21}$ These new technologies have been validated against the more traditional methods of microsatellite analysis and microdissection in other studies. $^{7,9,21,22}$ These results indicate that analysis of frozen or ethanol fixed samples is much less prone to PCR and other artifacts than the use of formalinfixed paraffin-embedded tissues. ${ }^{7,23}$ In addition, microsatellite analysis by fluorescent labeling and detection using automated systems is highly reliable and quantitative. ${ }^{8,10,11}$ However, the procedures used in the analysis of $\mathrm{LOH}$ are still under development and no established rules for statistical analysis currently exist. Several measures for LOH have been used, employing the mathematical mean and standard deviation of the allele ratios, newly developed scores for LOH by differential weighing of ratio results, either with or without rationalization for the various cutoff values employed. ${ }^{10,11,22}$

In the current study, we investigated and optimized each of the steps for microsatellite amplification analysis on DNA purified from blood lymphocytes. The main characteristics of our protocol are the rapid isolation of DNA followed by timerelease PCR amplification at single-molecule sensitivity, detection of the fluorescently labeled PCR products by capillary electrophoresis, and statistical assessment of AI via confidence intervals. Timerelease PCR has the advantage of minimizing manipulation steps associated with DNA purification and thus limiting the chances of loss of DNA or of DNA contamination. Since the PCR is set up as a single reaction, no intermediate PCR products need to be processed for nested PCR, which further reduces the risk of contamination and pipetting errors. In addition, intermediate PCR products may vary greatly in DNA content and may thus be prone to cross-sample contamination.

One crucial aspect of PCR analysis of DNA obtained from microdissected tissue is the amount of DNA that is present for amplification. When this amount falls below a critical level of around 50 alleles per PCR, the resulting $\mathrm{LOH}$ results become unstable. This phenomenon occurs in spite of the highly reliable and quantitative nature of fluorescent capillary electrophoresis detection and is the result of chance distribution of molecules in each amplification reaction. The amount of DNA that is present in the initial PCR may even be lower in the case of formalin-fixed tissue, where the DNA may be crosslinked or otherwise damaged. ${ }^{23}$ This level of damage cannot easily be quantified and may influence results even in the presence of apparently sufficient amounts of input DNA. ${ }^{7}$

In several different experiments the allele ratios obtained with near single-cell dilutions of DNA showed a chance distribution of the signals that was consistent with the presence or absence of one or the other allele in the reaction. We conclude from these results that the time-release PCR protocol is capable of amplifying single molecules of DNA. We also determined the reproducibility of the allele ratios with different markers. The signals obtained with larger alleles were generally lower than those obtained with the smaller alleles resulting in ratios below 1. A possible explanation for this phenomenon is that shorter alleles amplify slightly more effectively and therefore will contribute a larger amount of fluorescent signal in the ultimate analysis.

The average allele fraction observed from duplicate measurements on heterozygous lymphocyte DNA samples was remarkably similar across the seven microsatellite markers in this study. This result illustrates the highly quantitative capillary electrophoresis measurements across the set of di-, tri-, and pentanucleotide markers used in our study. Although the formulae presented for the calibration 
curve have tacitly assumed that a single measurement is made on each lymphocyte calibration sample and also on each sample from the tissue of interest, as we have illustrated, averages of multiple measurements on each sample can also be included. Such allele ratio measurements on multiple aliquots from a single subject's tissue sample will improve the precision of the estimates (both lymphocyte and tissue of interest). If the same number of measurements are made on every tissue sample the formulae above can validly be applied to the averaged values. If different numbers of aliquots are measured on different subjects or between lymphocyte (calibration) samples and samples of the tissue of interest, the principles of linear calibration can still be applied but the simple formulae given here are not adequate. Similarly, if the slope were believed to deviate from one and an estimate of the slope from a mixing experiment were employed, the formulae given here are not valid.

The decision as to whether or not a sample exhibits AI by determining whether a confidence interval covers a fixed value is in essence the result of a statistical test. One consequence of a testing viewpoint is that, instead of calculating a confidence interval for each tissue sample separately as we described, one could calculate critical values once and decide whether a sample showed evidence of AI by whether each $T\left(Y^{*}\right)$ fell below a lower critical value or above an upper one. A second consequence of the testing viewpoint is that the usual considerations about power and about multiple comparisons apply. Under the stated assumptions and for a given confidence coefficient $1-\alpha$, the power of this test depends on the unknown degree of AI that a sample contains, the variability among independent samples with the same level of imbalance (estimated by $s$ ) and on the number $(n)$ of lymphocyte samples in the calibration data. Of these factors, we have little control over the first or the second, which depends largely on the repeatability of the measurement procedure. We do control $n$, however, and can increase the power of the test to detect AI by increasing the number of subjects in the calibration sample.

We have presented a method for detecting AI based on calibration and illustrated its use. What we have left open is how many calibration curves are needed: one for each marker or even one for each heterozygous genotype within each marker? We do not have sufficient data now to answer these questions authoritatively. We currently recommend separate calibration curves for each marker although, with more data, the similarities evident Table 1 might be solidified and allow this requirement to be relaxed. The results in Table 1 are based on combining heterozygotes with different pairs of alleles. In many studies, the investigator will have too few heterozygotes with precisely the same alleles to develop separate calibration curves for each. Our preliminary data (not shown) suggested that the drift in apparent allele ratio as the size difference between the alleles grows was minimal, especially with a size difference smaller than 10 base pairs. We had limited data to examine this question, however, and would welcome additional investigations.

In conclusion, the use of time-release PCR using fluorescently labeled oligonucleotide primers followed by capillary electrophoresis provides a highly reliable method for the detection of AI when used on DNA of sufficient quantity and quality isolated from a small number of representative cells. Given the assumptions about heterozygosity of lymphocytes and about various statistical properties of the measurements of AI, a simple statistical procedure can be used to decide whether a given sample has AI. This method should prove valuable when large numbers of clinical specimens need to be investigated at multiple chromosomal loci.

\section{Acknowledgements}

We thank the principal investigator for the lung cancer screening study, Dr M Patricia Rivera, for her support of this project, and Elizabeth Long, Janet Archer, and Mark Bruno for logistical support.

\section{References}

1 Lengauer C, Kinzler KW, Vogelstein B. Genetic instabilities in human cancers. Nature 1998;396: 643-649.

2 Umar A, Kunkel TA. DNA-replication fidelity, mismatch repair and genome instability in cancer cells. Eur J Biochem 1996;238:297-307.

3 Coleman WB, Tsongalis GJ. The role of genomic instability in human carcinogenesis. Anticancer Res 1999;19:4645-4664.

4 Field JK, Liloglou T, Xinarianos G, et al. Genetic alterations in bronchial lavage as a potential marker for individuals with a high risk of developing lung cancer. Cancer Res 1999;59:2690-2695.

5 Sidransky D. Nucleic acid-based methods for the detection of cancer. Science 1997;278:1054-1059.

6 Hittelman WN. Clones and subclones in the lung cancer field. J Natl Cancer Inst 1999;91:1796-1799.

7 Euhus DM, Maitra A, Wistuba ii, et al. Use of archival fine-needle aspirates for the allelotyping of tumors. Cancer 1999;87:372-379.

8 Berg KD, Glaser CL, Thompson RE, et al. Detection of microsatellite instability by fluorescence multiplex polymerase chain reaction. J Mol Diagn 2000;2:20-28.

9 Moskaluk CA, Kern SE. Microdissection and polymerase chain reaction amplification of genomic DNA from histological tissue sections. Am J Pathol 1997;150: 1547-1552.

10 Liloglou T, Maloney P, Xinarianos G, et al. Sensitivity and limitations of high throughput fluorescent microsatellite analysis for the detection of allelic imbalance: application in lung tumors. Int J Oncol 2000;16:5-14.

11 Powell CA, Klares S, G OC, et al. Loss of heterozygosity in epithelial cells obtained by bronchial brushing: 
clinical utility in lung cancer. Clin Cancer Res 1999;5:2025-2034.

12 Liloglou T, Maloney P, Xinarianos G, et al. Cancerspecific genomic instability in bronchial lavage: a molecular tool for lung cancer detection. Cancer Res 2001;61:1624-1628.

13 Slebos RJ, Boerrigter L, Evers SG, et al. A rapid and simple procedure for the routine detection of ras point mutations in formalin-fixed, paraffin-embedded tissues. Diagn Mol Pathol 1992;1:136-141.

14 Westra WH, Baas IO, Hruban RH, et al. K-ras oncogene activation in atypical alveolar hyperplasias of the human lung. Cancer Res 1996;56:2224-2228.

15 Kebelmann-Betzing C, Seeger K, Dragon S, et al. Advantages of a new Taq DNA polymerase in multiplex PCR and time-release PCR. Biotechniques 1998;24:154-158.

16 Slebos RJ, Hoppin JA, Tolbert PE, et al. K-ras and p53 in pancreatic cancer: association with medical history, histopathology, and environmental exposures in a population-based study. Cancer Epidemiol Biomarkers Prev 2000;9:1223-1232.
17 Snedecor GW, Cochran WG. Statistical Methods, 8th edn. Iowa State University Press, USA, 1989. pp. 170-172.

18 Smith AL, Hung J, Walker L, et al. Extensive areas of aneuploidy are present in the respiratory epithelium of lung cancer patients. Br J Cancer 1996;73:203-209.

19 Wistuba ii, Lam S, Behrens C, et al. Molecular damage in the bronchial epithelium of current and former smokers. J Natl Cancer Inst 1997;89:1366-1373.

20 Wistuba ii, Behrens C, Milchgrub S, et al. Sequential molecular abnormalities are involved in the multistage development of squamous cell lung carcinoma. Oncogene 1999;18:643-650.

21 Simone NL, Bonner RF, Gillespie JW, et al. Laser-capture microdissection: opening the microscopic frontier to molecular analysis. Trends Genet 1998;14:272-276.

22 Wang Y, Hung SC, Linn JF, et al. Microsatellite-based cancer detection using capillary array electrophoresis and energy-transfer fluorescent primers. Electrophoresis 1997;18:1742-1749.

23 Williams C, Ponten F, Moberg C, et al. A high frequency of sequence alterations is due to formalin fixation of archival specimens. Am J Pathol 1999;155:1467-1471. 